\title{
Ultrasound B-Mode Imaging: Beamforming and Image Formation Techniques
}

\author{
Giulia Matrone ${ }^{1, *(D)}$, Alessandro Ramalli ${ }^{2,3, *(D)}$ and Piero Tortoli ${ }^{3, *} \mathbb{C}$ \\ 1 Department of Electrical, Computer and Biomedical Engineering, University of Pavia, 27100 Pavia, Italy \\ 2 Department of Cardiovascular Imaging and Dynamics, KU Leuven, 3000 Leuven, Belgium \\ 3 Department of Information Engineering, University of Florence, 50139 Florence, Italy \\ * Correspondence: giulia.matrone@unipv.it (G.M.); alessandro.ramalli@kuleuven.be (A.R.); \\ piero.tortoli@unifi.it (P.T.)
}

Received: 7 June 2019; Accepted: 16 June 2019; Published: 19 June 2019

check for updates

\section{Introduction}

In the last decade, very active research in the field of ultrasound medical imaging has brought to the development of new advanced image formation techniques and of high-performance systems able to effectively implement them [1]. For years, Brightness (B)-mode, one of the mostly used ultrasound imaging modalities [2], has been based on a time-consuming process, in which focused beams are iteratively sent into the body and the received waves are used to form an image scan-line, covering line-by-line the region of interest.

"Image formation" refers to the whole process of image reconstruction, starting from the transmission strategy to the reception of signals, beamforming, and image processing. The role of the so-called "beamformer" is central in this process, as it manages the ultrasound beam generation, focusing, and steering [3]. Image quality is in fact deeply influenced by the beam shape, and thus the beamforming optimization plays an important role in maximizing the signal-to-noise ratio, contrast, and resolution of the final image, while limiting as much as possible off-axis interferences to reject clutter and noise. Additionally, an important goal is to improve the acquisition frame-rate, which, as mentioned above, is limited by the line-by-line acquisition process [4].

Image enhancement methods play an important role during both the image pre- and post-processing phases [5]. In the former case, these techniques aim at improving the quality of B-mode frames by directly operating on the image generation process, as for example in spatial/frequency compounding, pulse compression, or harmonic imaging. The latter category instead refers to approaches aimed at reducing noise/artifacts, making speckle more uniform, detecting edges, and consequently facilitating the following processing steps, like segmentation or measurement of quantitative parameters.

Given the above premises, this Special Issue was launched to collect novel contributions on both ultrasound beamforming and image formation techniques. Twenty-one interesting works were consequently submitted and, among them, 10 were selected for publication (i.e., $48 \%$ acceptance rate).

\section{Ultrasound B-Mode Imaging}

The Special Issue opens with a review paper on the main ultrasound beamforming techniques [6]. The classic beamforming method for linear/phase array imaging is first introduced, before presenting advanced methods: from multi-line transmission and acquisition to synthetic aperture imaging, passing through plane wave, and diverging wave imaging. The stress is on the peculiarity of each method in terms of spatio-temporal resolution, contrast, penetration depth, aperture size, and field of view. The paper may represent a useful handbook for users who need to choose the most appropriate beamforming method for the specific application of interest. 
The following nine papers are grouped in three main groups, dealing with novel beamforming techniques, non-conventional image formation, and image enhancement, respectively.

\subsection{Novel Beamforming Techniques}

High frame-rate imaging techniques [4] have recently gained increased interest for their capability to detect fast dynamic events. However, the improvement of temporal resolution comes at the expense of image quality, thus pushing researchers to recover it by developing smart strategies. Four papers have been published in this Special Issue presenting advanced transmission sequences $[7,8]$ and beamforming schemes $[9,10]$ applied to either plane waves $[7,10]$ or multi-line transmission imaging $[8,9]$.

Bae and Song [7] analyzed the grating lobe artifacts due to the compounding of images obtained from the transmission of steered plane waves with a constant angle interval. Additionally, they showed that the use of non-uniform angle sets is a smart solution to keep the frame rate high, while limiting the level of image artifacts due to grating lobes. Tong et al. [8] studied the effectiveness of orthogonal coded excitations in multi-line transmission imaging in suppressing crosstalk artifacts. They showed that Golay codes enable higher crosstalk rejection (and better contrast) compared to linear chirps.

Two papers focus on the so-called coherence-based beamforming methods. Spatial coherence of ultrasound backscattered echoes is affected by contributions coming from off-axis regions, noise, and interferences. Matrone and Ramalli (Guest Editors) presented a new formulation of the Filtered Delay Multiply and Sum (F-DMAS) beamforming, namely Short-Lag F-DMAS [9]. They provided new insights into the relation between the performance of the F-DMAS algorithm and the coherence of backscattered signals in multi-line transmission imaging. Polichetti et al. presented a generalized and extended formulation of the F-DMAS beamformer, referred to as p-DAS [10]. They applied the proposed method to plane wave imaging and showed the achieved improvements in terms of lateral resolution and artifacts rejection.

\subsection{Non-Conventional Image Formation}

Non-conventional imaging systems have been proposed to improve the B-mode image quality and its diagnostic content. As an example, Inagaki et al. [11] designed and built a multi-modality (ultrasound and magnetic resonance) system to estimate the ultrasound propagation speed in the region of interest. The estimates were then used to correct the beamforming delay, both in transmission and in reception, thus enhancing the image resolution and signal-to-noise ratio. Liu et al. [12] proposed a multi-perspective ultrasound imaging system based on four $3.5 \mathrm{MHz}$ linear arrays. These arrays were placed, in a cross shape, on a motorized rotatory table to perform 3D ultrasound computed tomography of a breast model with different inclusions. The boundary of the breast, as well as the inclusions, could be clearly seen from all the perspectives, hence potentially improving the specificity and sensitivity of ultrasonic diagnosis.

\subsection{Image Enhancement}

Image quality enhancement can also be obtained through post-processing methods for image filtering, deconvolution, tracking, segmentation, and tissue characterization. In this Special Issue, Jabarulla and Lee [13] proposed a technique for liver images based on a signal reconstruction model, known as sparse representation over dictionary learning. This technique allows filtering the speckle while preserving the image features and the edges of anatomical structures. Guo et al. [14] presented a novel super-resolution reconstruction method. They developed a low computational load technique for microbubble localization and trajectory tracking. They showed that the proposed method improves the image resolution by using fewer frames than other reference methods, thus moving super-resolution a step forward to real-time imaging. Makūnaite et al. [15] showed how advanced segmentation and tracking techniques can be exploited to develop new predictors of cardiovascular events. Specifically, they tracked arterial wall movements for the evaluation of arterial stiffness and showed that the 
average value of the intima-media thickness, during the cardiac cycle, is statistically different between healthy volunteers and patients at risk of cardiovascular disease.

\section{Future Perspectives}

The different contributions published in this Special Issue confirm that the research of new strategies to improve the image formation process keeps on being a hot topic in the ultrasound imaging community. In this sense, it is also worth pointing out that efforts have been recently devoted to objectively evaluating and comparing novel beamforming methods, by creating development/test platforms and datasets $[16,17]$ to be shared by all research groups working on ultrasound beamforming.

Further active research is thus expected in this field, where many challenges still persist, especially when dealing with the difficult-to-image patients. For this reason, efforts should always be supported by real clinical needs, and image enhancement should be aimed at increasing visibility of anatomical structures and easing image interpretation and clinical parameters extraction, towards a more and more effective diagnostic process. An increasing involvement of clinicians in the in vivo evaluation of real image quality from a medical point of view is thus desirable.

Acknowledgments: The Guest Editors wish to thank all the authors who have submitted papers to this Special Issue and all the reviewers who allowed improving the quality of the submitted manuscripts by working with dedication and timeliness. Finally, we gratefully thank the editorial team of Applied Sciences and Daria Shi, our Assistant Editor, for their extraordinary support.

Conflicts of Interest: The authors declare no conflict of interest.

\section{References}

1. Boni, E.; Yu, A.C.H.; Freear, S.; Jensen, J.A.; Tortoli, P. Ultrasound Open Platforms for Next-Generation Imaging Technique Development. IEEE Trans. Ultrason. Ferroelectr. Freq. Control 2018, 65, 1078-1092. [CrossRef] [PubMed]

2. Szabo, T.L. Diagnostic Ultrasound Imaging: Inside Out, 1st ed.; Academic Press: Cambridge, MA, USA, 2004; ISBN 0-12-680145-2.

3. Van Veen, B.; Buckley, K.M. Wireless, Networking, Radar, Sensor Array Processing, and Nonlinear Signal Processing, 1st ed.; CRC Press: Boca Raton, FL, USA, November 2009; Volume Beamforming techniques for spatial filtering.

4. Tanter, M.; Fink, M. Ultrafast imaging in biomedical ultrasound. IEEE Trans. Ultrason. Ferroelectr. Freq. Control 2014, 61, 102-119. [CrossRef] [PubMed]

5. Contreras Ortiz, S.H.; Chiu, T.; Fox, M.D. Ultrasound image enhancement: A review. Biomed. Signal Process. Control 2012, 7, 419-428. [CrossRef]

6. Demi, L. Practical Guide to Ultrasound Beam Forming: Beam Pattern and Image Reconstruction Analysis. Appl. Sci. 2018, 8, 1544. [CrossRef]

7. Bae, S.; Song, T.-K. Methods for Grating Lobe Suppression in Ultrasound Plane Wave Imaging. Appl. Sci. 2018, 8, 1881. [CrossRef]

8. Tong, L.; He, Q.; Ortega, A.; Ramalli, A.; Tortoli, P.; Luo, J.; D’hooge, J. Coded Excitation for Crosstalk Suppression in Multi-Line Transmit Beamforming: Simulation Study and Experimental Validation. Appl. Sci. 2019, 9, 486. [CrossRef]

9. Matrone, G.; Ramalli, A. Spatial Coherence of Backscattered Signals in Multi-Line Transmit Ultrasound Imaging and Its Effect on Short-Lag Filtered-Delay Multiply and Sum Beamforming. Appl. Sci. 2018, 8, 486. [CrossRef]

10. Polichetti, M.; Varray, F; Béra, J.-C.; Cachard, C.; Nicolas, B. A Nonlinear Beamformer Based on p-th Root Compression-Application to Plane Wave Ultrasound Imaging. Appl. Sci. 2018, 8, 599. [CrossRef]

11. Inagaki, K.; Arai, S.; Namekawa, K.; Akiyama, I. Sound Velocity Estimation and Beamform Correction by Simultaneous Multimodality Imaging with Ultrasound and Magnetic Resonance. Appl. Sci. 2018, 8, 2133. [CrossRef]

12. Liu, C.; Zhang, B.; Xue, C.; Zhang, W.; Zhang, G.; Cheng, Y. Multi-Perspective Ultrasound Imaging Technology of the Breast with Cylindrical Motion of Linear Arrays. Appl. Sci. 2019, 9, 419. [CrossRef] 
13. Jabarulla, M.Y.; Lee, H.-N. Speckle Reduction on Ultrasound Liver Images Based on a Sparse Representation over a Learned Dictionary. Appl. Sci. 2018, 8, 903. [CrossRef]

14. Guo, W.; Tong, Y.; Huang, Y.; Wang, Y.; Yu, J. A High-Efficiency Super-Resolution Reconstruction Method for Ultrasound Microvascular Imaging. Appl. Sci. 2018, 8, 1143. [CrossRef]

15. Makūnaitè, M.; Jurkonis, R.; Rodríguez-Martínez, A.; Jurgaitienè, R.; Semaška, V.; Mèlinytė, K.; Kubilius, R. Ultrasonic Parametrization of Arterial Wall Movements in Low-and High-Risk CVD Subjects. Appl. Sci. 2019, 9, 465. [CrossRef]

16. Liebgott, H.; Rodriguez-Molares, A.; Cervenansky, F.; Jensen, J.A.; Bernard, O. Plane-Wave Imaging Challenge in Medical Ultrasound. In Proceedings of the 2016 IEEE International Ultrasonics Symposium (IUS), Tours, France, 18-21 September 2016; pp. 1-4.

17. Rodriguez-Molares, A.; Rindal, O.M.H.; Bernard, O.; Nair, A.; Bell, M.A.L.; Liebgott, H.; Austeng, A.; LØvstakken, L. The UltraSound ToolBox. In Proceedings of the 2017 IEEE International Ultrasonics Symposium (IUS), Washington, DC, USA, 6-9 September 2017; pp. 1-4.

(C) 2019 by the authors. Licensee MDPI, Basel, Switzerland. This article is an open access article distributed under the terms and conditions of the Creative Commons Attribution (CC BY) license (http://creativecommons.org/licenses/by/4.0/). 